\title{
Issues in implementing Knowledge Management
}

\author{
Narendra U P ${ }^{1}$, B S Pradeep ${ }^{2}$ \\ ${ }^{1}$ (Department of ISE, Mangalore Institute of Technology \& Engineering, Mangalore, India) \\ ${ }_{2}^{2}$ (Department of CSE, Rajarajeshwari College of Engineering, Bengaluru, India)
}

\begin{abstract}
Knowledge Management is playing a major role in shaping the Organization success. Knowledge management combines the ability of dealing with the data and information under the information technology. The major asset for any organization is the knowledge that any person posses. Knowledge management is used not just to store knowledge but also innovate and discover new knowledge. Knowledge can be of two types tacit and explicit. The knowledge that can be easily expressed and put on paper is known as explicit knowledge. The major concern in implementing KM is storing the tacit knowledge. Tacit refers to the knowledge that is inherent to any person. Also, the culture and organizational policies will play an important role in shaping up of the future of Knowledge management.
\end{abstract}

Keywords - codification, explicit, externalization, tacit

\section{INTRODUCTION}

The advent of technologies has changed the way in which we perceive knowledge. It is imperative for us to not just hoard information but make vital use of it in creating new knowledge. Knowledge Management (KM) has this become more and more relevant in today's information thrived world. IT has become very essential for every organization to manage knowledge effectively, thus gaining competitive advantage over others. Sharing knowledge is one of the most critical ways of building the organization future. Knowledge is an asset, which is very harder to be stored and managed, for the way in which it exists.

Knowledge, according to Nonaka [1] is a comprehensive concept with profound meanings, bearing the belief that it increases an organization's ability for effectual action. Knowledge is also defined as "justified true beliefs". Knowledge is derived from the information that we perceive. Information may exist in various forms, externally or internally. Knowledge relates to the way in which human analyses the information. For any organization, it is imperative to manage the knowledge to enhance its performance.

\section{KNOWLEDGE MANAGEMENT TECHNOLOGY}

Knowledge management is a process of managing knowledge by creating, sustaining, applying, sharing and renewing knowledge to enhance organizational performance and create values [2]. This involves both human responsibility as well as the role of information technology (IT). The vast growth that is witnessed in the IT has provided more flexibility in managing the information. KM involves not just storing and retrieving knowledge. It involves more of human decisions in analyzing the huge repository of stored knowledge. The major knowledge management technologies include data mining, case-based reasoning, information retrieval, topic maps, weblogs, e-portfolio [3].

The data mining helps a student to select information from the available huge repository; improving the efficiency of identifying interesting information objects. The case based reasoning helps students to learn much more from the old cases; doing benefit to them when developing innovative technologies or learning new knowledge. Learners can retrieve new information and make information more explicit through Information retrieval technologies. The Topic maps provide reflective learning to the students; being useful for raising questions and analyzing; helping students to master comprehensive knowledge. The students can learn with peers and make great progress via Weblogs. And the E-portfolios deepen the learning and help students to master comprehensive knowledge.

Knowledge can be classified into two categories: explicit and tacit [4]. Explicit knowledge is something that can be well articulated. It can be put on paper, expressed on some media, explained by some means. For ex: a manual on operating a television. Tacit knowledge is something that cannot be well articulated. It is the knowledge that is internal to a human, which is difficult to be expressed. It is difficult to express, write down or transfer tacit knowledge to other media. Tacit knowledge is more of 'know-how' knowledge. For ex: How to swim.

Tacit knowledge is the knowledge that a person possesses, and will be of value to the organization. The complexity in externalizing this makes the process of knowledge management more difficult. Tacit knowledge is to be converted into explicit through interactions among the knowledge repositories. The process of knowledge transfer seems relative simple but there are lots of hindrances in it. Tacit knowledge may exist in 
various forms. It may be individual or collective [5]. Tacit knowledge may be the knowledge acquired through different means. It may be the knowledge that a person has acquired through his experience, but may not be able to express in words or put on paper.

Tacit knowledge can be the learning acquired collectively in an organization as part of the work that a person is involved. This may be an individual learning or learning acquired collectively in group by means of knowledge sharing amongst them. For ex. few of the best practices shared in accomplishing a work. For any organization, it will need the tacit knowledge of a resource person to be stores, so that it can be shared later with other people. The goal of KM is to effectively externalize both the types of knowledge.

\section{EXTERNALIZATION OF TACIT KNOWLEDGE}

As the tacit knowledge is inherent to a person, we need to look at different ways of decoding the information. Few techniques that can be used are - interviews, discussion forums, weblogs, and concept maps. The resource person can be interviewed on the topic of his expertise, by which we should try to extract and store the inherent knowledge. Group discussions on areas of expertise can be conducted to capture the inherent knowledge. The retrieved knowledge should be stored in the knowledge bank. The knowledge shared by the resource person remains with the organization permanently. The knowledge stored will be part of the knowledge bank, even if the person leaves the organization.

Concept maps is the another technique that can be used to store the tacit knowledge. Concept map is a hierarchical way of organizing concepts. It uses graphical tools for organizing and representing knowledge [6]. It represents the cognitive structure of the human brain. Expressing knowledge using concept map is a creative way of organizing information. Concept maps are constructed with reference to a question, known as a focus question. Accordingly concepts are arranged hierarchically with the most general concept at the top and more specific below. In this context, we can identify focus questions for different scenarios and build concept maps that would be easy to understand to the learner.

The process of managing the tacit knowledge involves - knowledge assimilation, knowledge storage and knowledge retrieval. The process is show in Fig 1The initial process of assimilation of knowledge involves - how to incorporate the integral tacit knowledge onto the knowledge repository. As discussed, we can include the various techniques like Interviews, discussion forums, Course maps etc. The decoded information has to be stored in the repository and formatted to suit our future needs. The knowledge cannot be stores in the as-is format. Rather we may need to structure it so make it adaptable to other technologies. The knowledge stored has to be indexed, to make it compatible to be used with various search and recommender technologies.

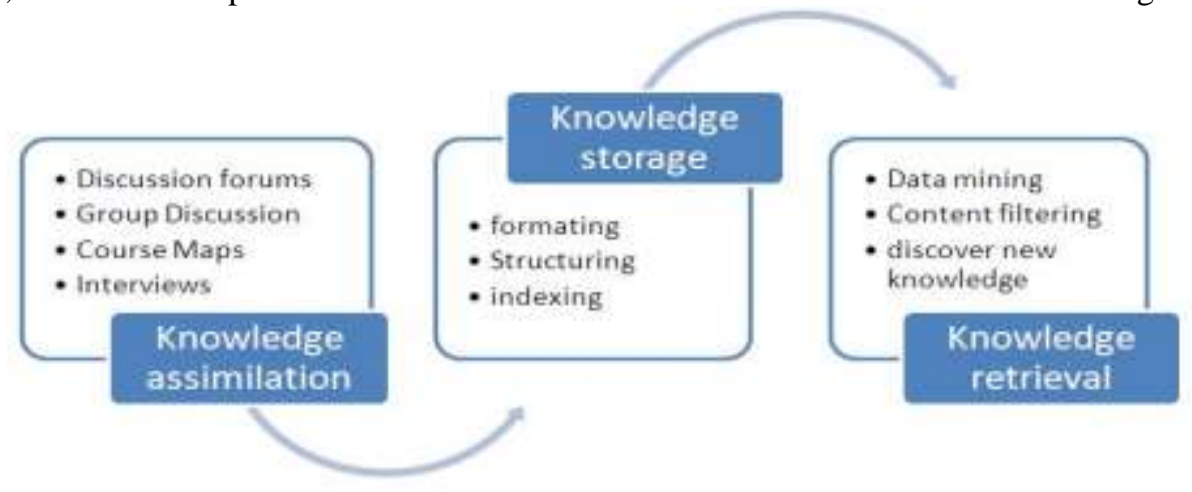

Fig 1 Managing Tacit knowledge

The last stage of the managing process involves knowledge retrieval techniques. The knowledge stored needs to be retrieved in an efficient way. To support this, techniques like data mining, content filtering and recommender systems can be used. A knowledge repository should not just act as a storage tool, but also should aid in extracting new information from the existing ones. One of the important goals of KM is to discover new knowledge from the stored knowledge.

\section{ISSUES IN EXTERNALIZATION}

The process of externalization of tacit knowledge is known as codification. The same can be visualized as in Fig 2. The process of codification involves lot of issues. Most of the knowledge that a person may possess may not be easily codified. It may not be possible to express everything that a person knows. In such cases, codification may not happen. Many will have an inhibition to share their knowledge. It is important for a resource person to share his knowledge. The Organization culture should also support the knowledge sharing. Many people may fear that, by sharing the knowledge, their position in the organization may be insecure. The 
Organization culture is one of the major reasons for this. If there is a healthy environment in an organization, people may not fear to share their knowledge.

Resource person will always look at the benefit in sharing. The lack of individual benefit in sharing will deter them in externalizing the knowledge. Organizations should not just support, but also provide benefits in the process. The knowledge shared will benefit less to the resource person, but will be a major asset to the organization in the longer run. Whether the resource person stays in the organization or not, anytime in the future, the knowledge shard will help the future employees who are working on the same area. This should be the considering factor for the organization to provide motivating factors to the resource persons.

The technology used for the codification is only few suggested approaches, but not a standard process. It does not guarantee the success of externalization. The success depends on the person involved. There is no suitable technology to externalize knowledge. The interviews, discussions, with the resource person will yield us raw information on a particular subject. But it is essential to format and structure it to suit the KM architecture. The knowledge extracted will be of use, only if it can be retrieved and made use of in any future context. Standard process has to be followed to ensure the standardization of KM repository.

\section{CONCLUSION}

Knowledge is the biggest asset for any organization. For any organization to thrive in the business, it is important not just to use the knowledge of a person, but also look at ways in which the same can be stored and used for future use. KM technologies is now been considered as the major technique that can be used to store knowledge. KM also aids in retrieving new knowledge from the existing one. The major hurdle in implementing $\mathrm{KM}$ is the people mindset, organization policies and lack of standard process. Organization should value the resources and provide right type of motivation, which will push the resource persons to share their knowledge amongst their co workers. Healthy environment in the organization should be established to aid the same. The process of coding and structuring of knowledge in the KM tool will always be another issue in the implementation process. Without any standard tools or practices, externalization will never succeed. Positive individual mindset and Organization policies can ensure the success of KM implementation.

\section{REFERENCES}

[1] Nonaka I, A dynamic theory of Organizational knowledge creation, Organization Science, 5(1), 1994, 14-37.

[2] Wu Kebao, Dai Junxum,, Knowledge management technologies in education, International symposium on knowledge acquisition and modeling, 2008.

[3] Marc Henselewski, Evaluation of knowledge management technologies for the support of technology forecasting, proceedings of the $39^{\text {th }}$ Hawaii international conference on system sciences, 2006

[4] M Polanyi, The tacit dimension (Doubleday \& Company, Inc, Garden City, New York, 1966)

[5] J Casstillo, A note on the concept of tacit knowledge, Journal of Management Inquiry, vol 11, Mar 2002, pp 46-57

[6] Novak J D, A J Canas, The theory underlying Concept Maps and how to construct and use them, Technical report on IHMC CMap tools 2006-0 Rev 2008-01, Florida institute for Human and machine cognition, 2008 